\title{
Preface to special issue on "Peripheral Nerve Repair and Regeneration"
}

\section{Francesca Toia}

Plastic and Reconstructive Surgery, Department of Surgical, Oncological and Oral Sciences, University of Palermo, 90127 Palermo, Italy.

Address for correspondence: Dr. Francesca Toia, Plastic and Reconstructive Surgery, Department of Surgical, Oncological and Oral Sciences, University of Palermo, 90127 Palermo, Italy. E-mail: francescatoia@gmail.com

Peripheral nerve surgery has achieved a great improvement in the last century. The introduction of microsurgery and the technical advances in reparative and reconstructive techniques has been instrumental in advancing nerve surgery techniques. Yet, in the last few decades, the scientific progress has slowed down significantly, and surgery is still far from reaching an optimal functional recovery in most cases.

New surgical approaches are increasingly used for various indications, but current research mainly focuses on the mechanisms of nerve damage and nerve regeneration. It is now clear that nerve regeneration not only relies on surgical reconstruction but also on understanding underlying biomolecular processes that could turn out to be the key for developing novel treatment strategies.

With the present special issue on "nerve regeneration and repair", we wish to summarize the state of the art of translational and clinical research and present the current trends and future prospects in peripheral nerve surgery. For this purpose, most of the twelve papers in this issue are review papers.

This issue begins with an overview of the current neurophysiologic and imaging tests: preoperative diagnostic work-up and postoperative monitoring, to provide a clinical guide on the assessment of nerve injuries.

Then, we discuss nerve pain and dysfunction following surgery (e.g. in scar neuropathy or in recalcitrant compression neuropathy) and the treatment approaches. Using current literature, we summarize the analysis of reasons for treatment and the current clinical and surgical

\begin{tabular}{|l|l|}
\hline \multicolumn{2}{|c|}{ Access this article online } \\
\hline Quick Response Code: & Website: \\
\hline & www.parjournal.net \\
\hline & \\
\hline & \\
\hline
\end{tabular}

recommendations. Three papers of this issue focus on different aspects of nerve pain, and suggest promising directions for research on the mechanism of nerve regeneration and nerve guidance (e.g. investigation of genetics and biochemical signaling) and novel therapeutic approaches (e.g. neurostimulation).

We also reviewed modern advances in surgical techniques for complex nerve injuries, such as vascularized nerve grafts, which are indicated for long nerve gaps and scarred beds, and nerve transfers, which are indicated for proximal nerve injuries.

Free vascularized nerve grafts were first described in the 1970s. After initial enthusiasm, their popularity decreased partly due to their technical difficulty, and only few surgeons used the technique. Yet, they perform better than nonvascularized nerve grafts and provide advantage in recovery in selected cases. Their potential could find a greater expression in the next future, as discussed in a review article.

Nerve transfers have opened new horizons in nerve repair strategies: first described in the 19 th century, they have revolutionized the 21th century approach to nerve injuries, particularly proximal injuries. They are a valuable tool for otherwise unrepairable nerve lesions candidates to palliative treatment (e.g. tendon transfer) and are finding increasing indications for repair of both motor and sensory nerves. Current indications in the upper limb nerve injuries are reviewed. Also, two of the papers in this issue are focused on "sensory protection" and "babysitting procedures": local nerves can be redirected to a distal target to prevent the muscle atrophy and the functional loss that follows prolonged denervation.

To complete the tableau of future prospects in nerve regeneration, two papers of this special issue are focused on two novel fields of research: tissue-engineered conduits and robotic-assisted microsurgery.

Ongoing research holds the potential of revolutionizing our approach to nerve repair and regeneration. Tissue-engineering investigates the potential of different biomimetic materials as peripheral nerve scaffold, and 
modifies and directs interactions between cells, growth factors and signaling molecules, and biomaterials, to guide nerve regeneration. Robotic-assisted microsurgery represents a great technological advance, which can be further developed for specific applications in peripheral nerve surgery. It allows a minimally invasive approach, reducing morbidity and perineural adherences and favoring a better nerve regeneration.

Lastly, we looked at composite tissue allotransplantation, where nerve regeneration holds specific features, as the host interaction with allogenic tissues and the need for immunosuppression; the last paper of this issue discusses the role of cortical reorganization, drugs (such as tacrolimus) and adipose-derived stem cells for axonal regeneration and myelination.
I hope that the papers presented in this special issue will serve as a reference and inspiration for students, researchers, and clinicians who have interest in nerve surgery.

Thanks to all the authors and the reviewers for their contributions and to the editorial staff of PAR, for working on this special issue and for their precious and continuous support.

I hope you enjoy reading this special issue.

How to cite this article: Toia F. Preface to special issue on "Peripheral Nerve Repair and Regeneration". Plast Aesthet Res 2015;2:147-8.

Source of Support: Nil, Conflict of Interest: None declared.

Received: 07-04-2015; Accepted: 20-05-2015 